\title{
CORRECTION
}

\section{Correction to: Incidence of severe sepsis and septic shock in German intensive care units: the prospective, multicentre INSEP study}

Gernot Marx* and SepNet Critical Care Trials Group

(c) 2017 Springer-Verlag GmbH Germany and ESICM

\section{Correction to: Intensive Care Med (2016) \\ 42:1980-1989 \\ DOI 10.1007/s00134-016-4504-3}

The members of the SepNet Critical Care Trials Group were provided in such a way that they could not be indexed as collaborators on PubMed. The publisher apologizes for this error and is pleased to list the members of the group here:

\begin{tabular}{llll}
\hline First name & Last name & City & Country \\
\hline Jens & Litmathe & Aachen & Germany \\
Jörg B. & Schulz & Aachen & Germany \\
Manuel & Dafotakis & Aachen & Germany \\
Thomas & Möllhoff, M.Sc. & Aachen & Germany \\
Carolin & Stalljohann & Aachen & Germany \\
Jonas & Engels & Aachen & Germany \\
Barbara & Sauerzapfe & Aachen & Germany \\
Barbara & Stolzenberg & Aachen & Germany \\
Engels & Jonas & Aachen & Germany \\
Thomas & Möllhoff & Aachen & Germany \\
Stalljohann & Carolin & Aachen & Germany \\
Beeker & Thorben & Aachen & Germany \\
Marx & Gernot & Aachen & Germany \\
Schäfer & Melanie & Aachen & Germany \\
Schürholz & Tobias & Aachen & Germany \\
Altiok & Ertunc & Aachen & Germany \\
Nikolaus & Marx & Aachen & Germany \\
Kersten & Alexander & Aachen & Germany \\
\hline
\end{tabular}

*Correspondence: gmarx@ukaachen.de

Department of Intensive Care Medicine, University Hospital RWTH

Aachen, c/o G. Marx, Pauwelsstr. 30, 52074 Aachen, Germany

The online version of the original article can be found under https://doi. org/10.1007/s00134-016-4504-3.

\section{Springer}

\begin{tabular}{|c|c|c|c|}
\hline First name & Last name & City & Country \\
\hline Dückers & Hannah & Aachen & Germany \\
\hline Koch & Alexander & Aachen & Germany \\
\hline Christian & Trautwein & Aachen & Germany \\
\hline Alexander & Scharf & Altenburg & Germany \\
\hline Ilse & Kreuzer & Augsburg & Germany \\
\hline Ulrich & Jaschinski & Augsburg & Germany \\
\hline Ilse & Kreuzer & Augsburg & Germany \\
\hline Ulrich & Jaschinski & Augsburg & Germany \\
\hline Markus & Niklas & Bad Tölz & Germany \\
\hline Michael & Kulzer & Bad Tölz & Germany \\
\hline Martin & Schlott & Bad Tölz & Germany \\
\hline Thomas & Iberer & Baden-Baden & Germany \\
\hline Oelert & Stella & Bergisch Gladbach & Germany \\
\hline Löhr & Thorsten & Bergisch Gladbach & Germany \\
\hline Pillai & Vandana & Bergisch Gladbach & Germany \\
\hline Damaris & Praeger & Berlin & Germany \\
\hline Fabian & Knebel & Berlin & Germany \\
\hline Gert & Baumann & Berlin & Germany \\
\hline Simone & Rosseau & Berlin & Germany \\
\hline Florian & Schlenk & Berlin & Germany \\
\hline Bodo & Hoffmeister & Berlin & Germany \\
\hline Friederike & Compton & Berlin & Germany \\
\hline Clemens & Hoffmann & Berlin & Germany \\
\hline Sibylle & Rademacher & Berlin & Germany \\
\hline Michael & Vietzke & Berlin & Germany \\
\hline Achim & Jörres & Berlin & Germany \\
\hline
\end{tabular}




\begin{tabular}{|c|c|c|c|}
\hline First name & Last name & City & Country \\
\hline Andreas & Meisel & Berlin & Germany \\
\hline Benjamin & Hotter & Berlin & Germany \\
\hline Annerose & Mengel & Berlin & Germany \\
\hline Anton & Goldmann & Berlin & Germany \\
\hline Frank & Nöther & Berlin & Germany \\
\hline Didier & Keh & Berlin & Germany \\
\hline Katrin & Schmidt & Berlin & Germany \\
\hline Stefan & Angermair & Berlin & Germany \\
\hline Magnus & Kaffarnik & Berlin & Germany \\
\hline Florian & Bubser & Berlin & Germany \\
\hline Immo & Gummelt & Bielefeld & Germany \\
\hline Ina & Vedder & Bielefeld & Germany \\
\hline Friedhelm & Bach & Bielefeld & Germany \\
\hline Markus & Winter & Blaubeuren & Germany \\
\hline Frank & Weißer & Blaubeuren & Germany \\
\hline Marc & Roederer & Blaubeuren & Germany \\
\hline Stefan & Martini & Bochum & Germany \\
\hline Lars & Bergmann & Bochum & Germany \\
\hline Michael & Adamzik & Bochum & Germany \\
\hline Andreas & Baumann & Bochum & Germany \\
\hline Britta & Wolf & Bochum & Germany \\
\hline Peter K. & Zahn & Bochum & Germany \\
\hline Marcus & Lehnhardt & Bochum & Germany \\
\hline Adrien & Daigeler & Bochum & Germany \\
\hline Andreas Hohn & Andreas & Bochum & Germany \\
\hline Katrin & Reinhold & Bochum & Germany \\
\hline Michael & Adolf & Bochum & Germany \\
\hline Nicole & Altenbeck & Bochum & Germany \\
\hline Christoph & Gussone & Bochum & Germany \\
\hline Johannes Wolfgang & Dietrich & Bochum & Germany \\
\hline Roland & Köditz & Bochum & Germany \\
\hline Christian & Putensen & Bonn & Germany \\
\hline Jens-Christian & Schewe & Bonn & Germany \\
\hline Thomas & Muders & Bonn & Germany \\
\hline Jens & Soukup & Cottbus & Germany \\
\hline Kornel & Skitek & Cottbus & Germany \\
\hline Wolfgang & Geisser & Dillingen an der Donau & Germany \\
\hline Andreas & Alber & Dillingen an der Donau & Germany \\
\hline Ludwig & Düthorn & Donauwörth & Germany \\
\hline Josef & Demund & Donauwörth & Germany \\
\hline Ludwig & Düthorn & Donauwörth & Germany \\
\hline Isabel & v. Criegern & Donauwörth & Germany \\
\hline Andreas & Nowak & Dresden & Germany \\
\hline Toralf & Morgenstern & Dresden & Germany \\
\hline Christoph & Gliniorz & Dresden & Germany \\
\hline Michael & Meisner & Dresden & Germany \\
\hline Melanie & Dexel & Dresden & Germany \\
\hline Jan Karl & Schütte & Düren & Germany \\
\hline Markus H. & $\begin{array}{c}\text { Huppertz- } \\
\text { Thyssen }\end{array}$ & Düren & Germany \\
\hline Stefan & Schröder & Düren & Germany \\
\hline
\end{tabular}

\begin{tabular}{|c|c|c|c|}
\hline First name & Last name & City & Country \\
\hline Ralf & Kowalzik & Düren & Germany \\
\hline Cansu & Guel & Düren & Germany \\
\hline Rainer & Terhorst & Düren & Germany \\
\hline Peter & Nießen & Düren & Germany \\
\hline Rolf & Hauschild & Eisenach & Germany \\
\hline Elisabeth-Maria & Mutzek & Eisenach & Germany \\
\hline Klaus & Kogelmann & Emden & Germany \\
\hline Matthias & Drüner & Emden & Germany \\
\hline Dominik & Jarczak & Emden & Germany \\
\hline Christian & Forster & Erlangen & Germany \\
\hline Carsten & Willam & Erlangen & Germany \\
\hline K.U. & Eckardt & Erlangen & Germany \\
\hline Rüdiger & Clemenz & Erlangen-Nürnberg & Germany \\
\hline Sven & Siemonsen & Erlangen-Nürnberg & Germany \\
\hline Matthias & Pauschinger & Erlangen-Nürnberg & Germany \\
\hline Matthias & Baumgärtel & Erlangen-Nürnberg & Germany \\
\hline Dorothea & Muschner & Erlangen-Nürnberg & Germany \\
\hline Michael & Christ & Erlangen-Nürnberg & Germany \\
\hline Martin & Wenzl & Erlangen-Nürnberg & Germany \\
\hline Rainer & Höhl & Erlangen-Nürnberg & Germany \\
\hline Axel & Junger & Erlangen-Nürnberg & Germany \\
\hline Armin & Wöhrle & Esslingen & Germany \\
\hline Henry & Kanthak & Esslingen & Germany \\
\hline Matthias & Leschke & Esslingen & Germany \\
\hline Henry & Kanthak & Esslingen & Germany \\
\hline Armin & Wöhrle & Esslingen & Germany \\
\hline Matthias & Leschke & Esslingen & Germany \\
\hline Paul & Kessler & Frankfurt & Germany \\
\hline Jochen & Souquet & Frankfurt & Germany \\
\hline Andreas & Holfeld & Frankfurt & Germany \\
\hline Oliver & Habler & Frankfurt & Germany \\
\hline Markus & Thörner & Frankfurt & Germany \\
\hline Oliver & Vogt & Frankfurt & Germany \\
\hline Patrick & Meybohm & Frankfurt am Main & Germany \\
\hline Kai & Zacharowski & Frankfurt am Main & Germany \\
\hline Simone & Lindau & Frankfurt am Main & Germany \\
\hline Marcus & Lenk & Fürstenfeldbruck & Germany \\
\hline Florian & Weis & Fürstenfeldbruck & Germany \\
\hline Hermann & Schubert & Fürstenfeldbruck & Germany \\
\hline Jürgen & Kemnitz & Geilenkirchen & Germany \\
\hline Sven & Reckort & Geilenkirchen & Germany \\
\hline Martina & Weikert & Geilenkirchen & Germany \\
\hline Ulrich & Sudhues & Geilenkirchen & Germany \\
\hline Stefan & Möller & Geilenkirchen & Germany \\
\hline Pinger & Stefan & Geilenkirchen & Germany \\
\hline Andrea & Domke & Gelsenkirchen & Germany \\
\hline Christoph & Wiesenack & Gelsenkirchen & Germany \\
\hline Florian & Schmidt & Gelsenkirchen & Germany \\
\hline Kai-Uwe & Döbel & Gotha & Germany \\
\hline Onnen & Moerer & Göttingen & Germany \\
\hline Jörn & Schäper & Göttingen & Germany \\
\hline
\end{tabular}




\begin{tabular}{|c|c|c|c|c|c|c|c|}
\hline First name & Last name & City & Country & First name & Last name & City & Country \\
\hline Andrea & Kernchen & Göttingen & Germany & Henning & Krep & Köln & Germany \\
\hline Matthias & Gründling & Greifswald & Germany & Helge & Anders & Köln & Germany \\
\hline Jan & Bollmann & Greifswald & Germany & Sanjay & Aduckathil & Köln & Germany \\
\hline Sven-Olaf & Kuhn & Greifswald & Germany & Samir & Sakka & Köln-Mehrheim & Germany \\
\hline Gregor & Kemming & Günzburg & Germany & Jan Hinnerk & Reessing & Köln-Mehrheim & Germany \\
\hline Carsten & Einfeld & Günzburg & Germany & Sabrina & Aabaid & Köln-Mehrheim & Germany \\
\hline Thomas & Sommerer & Günzburg & Germany & Mathias & Löbe & Leipzig & Germany \\
\hline Gregor & Kemming & Günzburg & Germany & Holger & Bogatsch & Leipzig & Germany \\
\hline Carsten & Einfeld & Günzburg & Germany & Marcus & Löffler & Leipzig & Germany \\
\hline Thomas & Sommerer & Günzburg & Germany & Christoph & Engels & Leipzig & Germany \\
\hline Philipp & Köntges & Güstrow & Germany & Gerd Peter & Molter & Leverkusen & Germany \\
\hline Burkhard & Hinz & Güstrow & Germany & Michael & Stunz & Leverkusen & Germany \\
\hline Lars-Oliver & Jauch & Güstrow & Germany & Anja & Mitrenga & Leverkusen & Germany \\
\hline Lutz & Selonke & Güstrow & Germany & Götz & Geldner & Ludwigsburg & Germany \\
\hline Stefan & Kluge & Hamburg & Germany & Monica & Bürle & Ludwigsburg & Germany \\
\hline Axel & Nierhaus & Hamburg & Germany & Robert & Klasen & Lünen & Germany \\
\hline Geraldine & de Heer & Hamburg & Germany & Steffanie & Werner & Lünen & Germany \\
\hline Marco & Gruß & Hanau & Germany & Wolfram & Wilhelm & Lünen & Germany \\
\hline Katja & Köhler & Hanau & Germany & Frenzel & Silke & Mühlhausen & Germany \\
\hline Alexander & Brinkmann & Heidenheim & Germany & Illert & Juliane & Mühlhausen & Germany \\
\hline Thomas & Fuchs & Heidenheim & Germany & Schuttpelz & Jana & Mühlhausen & Germany \\
\hline Andreas & Köberer & Heidenheim & Germany & Josef & Briegel & München & Germany \\
\hline Markus & Geuting & Heilbronn & Germany & Patrick & Möhnle & München & Germany \\
\hline Henry & Weigt, MBA & Heilbronn & Germany & Adrian & Knorr & München & Germany \\
\hline Thomas & Baltus & Heinsberg & Germany & Wolfgang & Hartl & München & Germany \\
\hline Claudia & Scheltz & Hohenfelde & Germany & Ludwig & Ney & München & Germany \\
\hline Frank & Bloos & Jena & Germany & Hans-Joachim & Stemmler & München & Germany \\
\hline Konrad & Reinhart & Jena & Germany & Hans-Walter & Pfister & München & Germany \\
\hline Rudolf & Jegen & Jülich & Germany & Matthias & Angstwurm & München & Germany \\
\hline Marcus & Flucht & Jülich & Germany & Lorenz & Frey & München & Germany \\
\hline Gburek & Jürgen & Jülich & Germany & Michael & Irlbeck & München & Germany \\
\hline Marcus & Flucht & Jülich & Germany & Volker & Huge & München & Germany \\
\hline Rudolf & Jegen & Jülich & Germany & Marion & Weis & München & Germany \\
\hline Anne & Stumm & Kempten & Germany & Erich & Kilger & München & Germany \\
\hline Jannik & Rossenbach & Kempten & Germany & Klaus & Hofmann-Kiefer & München & Germany \\
\hline Anne & Stumm & Kempten & Germany & Thomas & Felbinger & München & Germany \\
\hline Mathias & Haller & Kempten & Germany & Ines & Kaufmann & München & Germany \\
\hline Anne & Stumm & Kempten & Germany & Julia & Goeschl & München & Germany \\
\hline Jannik & Rossenbach & Kempten & Germany & Franz & Brettner & München & Germany \\
\hline John & Lynch & Koeln & Germany & Heiner & Hamm & München & Germany \\
\hline Martin & Kempkens & Koeln & Germany & Claudia & Eife & München & Germany \\
\hline Volkmar & Buechner & Koeln & Germany & Claus & Peckelsen & Münschen & Germany \\
\hline Dieter & Molitor & Köln & Germany & Michael & Findeisen & Münschen & Germany \\
\hline Gerda & Klasen & Köln & Germany & Patrick & Schmidt- & Münschen & Germany \\
\hline Bruno & Rahmen & Köln & Germany & & Schridde & & \\
\hline Petra & Muhr & Köln & Germany & Andreas & Kuner & Nagold & Germany \\
\hline Patricia & Canaviri & Köln & Germany & Thomas & Walz & Nagold & Germany \\
\hline Fritz & Fiedler & Köln & Germany & Barbara & Kuner & Nagold & Germany \\
\hline Jürgen & Lutz & Köln & Germany & Thomas & Schrauzer & Nürnberg & Germany \\
\hline Marc & Kaiser & Köln & Germany & Jens & Nentwich & Nürnberg & Germany \\
\hline Simon & Klünter & Köln & Germany & Stefan & John & Nürnberg & Germany \\
\hline
\end{tabular}




\begin{tabular}{llll}
\hline First name & Last name & City & Country \\
\hline Christian & Byhahn & Oldenburg & Germany \\
Thomas & Kretschmer & Oldenburg & Germany \\
\hline Martin N. & Bergold & Oldenburg & Germany \\
\hline Andreas & Weyland & Oldenburg & Germany \\
Florian & Jelschen & Oldenburg & Germany \\
Anja & Diers & Oldenburg & Germany \\
Frieder G. & Knebel & Pößneck & Germany \\
Thomas & Kupfer & Pößneck & Germany \\
Michael & Oppert & Potsdam & Germany \\
Steffen & Rohde & Potsdam & Germany \\
Angela & Möllemann & Radebeul & Germany \\
Uwe & Oehmichen & Radebeul & Germany \\
Theresa & Renner & Radebeul & Germany \\
Hans-Georg & Bone & Recklinghausen & Germany \\
Jörg & Freyhoff & Recklinghausen & Germany \\
Dana & Holst & Ribnitz-Damgarten & Germany \\
Thomas & Birken & Rostock & Germany \\
Jörg & Henschel & Rostock & Germany \\
Martin & Gloger & Rostock & Germany \\
Peter & Brock & Rostock & Germany \\
Stephan & Klösel & Rüsselsheim & Germany \\
Gerd & Albuszies & Rüsselsheim & Germany \\
Detlef & Schumacher & Schwerin & Germany \\
Rainer & Meierhenrich & Stuttgart & Germany \\
\hline & & &
\end{tabular}

\begin{tabular}{llll}
\hline First name & Last name & City & Country \\
Alexej & Schöpp & Stuttgart & Germany \\
Harald & Kuhn & Stuttgart & Germany \\
Reimer & Riessen & Tübingen & Germany \\
Michael & Haap & Tübingen & Germany \\
Silvia & Ziegler & Tübingen & Germany \\
Jana & Fastnacht- & Ueckermünde & Germany \\
& Böttcher & & \\
Rolf Jürgen & Schröder & Ueckermünde & Germany \\
Manfred & Weiss & Ulm & Germany \\
Katrin & Frank & Ulm & Germany \\
Michael & Georgieff & Ulm & Germany \\
Manfred & Weiss & Ulm & Germany \\
Eberhard & Barth & Ulm & Germany \\
Henrik & Bracht & Ulm & Germany \\
Heidrun & Ehmcke & Waren & Germany \\
Astrid & Francke & Waren & Germany \\
Stefan & Ott & Waren & Germany \\
Jens & Köpcke & Wismar & Germany \\
Andreas & Meyer & Wismar & Germany \\
Gunnar & Jokiel & Wolgast & Germany \\
Thomas & Freund & Würselen & Germany \\
Guido & Schick & Würselen & Germany \\
Evagelos & Karassimos & Würselen & Germany \\
\hline & & & \\
\hline
\end{tabular}

Published online: 1 December 2017 